\title{
Game Learning Analytics Applied to the Use of Instant Messaging Apps and Discussion Forums in Online Higher Education
}

\begin{abstract}
With the growth of virtuality and digital education, students increasingly interact in a variety of ways. The potential effects of these interactions on their learning process are not fully understood and the outcomes may depend on the tool used for the interaction. This study explores the communication patterns and learning effectiveness acquired by students using two basic synchronous and asynchronous communication tools in e-learning environments, specifically business simulation games. We conduct an experiment with 478 online business students, 267 of whom used online discussion forums while the remaining 211 interacted via an instant messaging app during their participation in the game. The application of learning analytics and data mining on natural language processing allows us to explore the student communication patterns with each of tools and their effectiveness in terms of learning. Our results confirm the complementarity of the communication tools, asynchronous tools being especially the suitable for task-related communication and synchronous ones for speeding up and facilitating student interactions. The contribution of our research is two-fold. On one hand, this study reveals the utility of game learning analytics applied to online communication in better understanding the learning process of online students participating in games. On the other hand, this study highlights several lessons for the online learning agents, educational institutions, teachers, and students. These include evidence-based recommendations for implementing better communication tools in online education, and a greater understanding of the learning outcomes achieved.
\end{abstract}

Keywords: online messaging apps; online discussion forums; game learning analytics; data mining higher education.

\section{Introduction}

Virtuality has recently emerged as a new paradigm at education, changing and enhancing teaching and learning possibilities. Digital education, understood as current online educational practices, is projected to surpass 243 billion U.S. dollars worldwide by 2022 (The Statistics Portal, 2017). It has growing support from teachers and students who show their willingness to support digital education models and embrace digital learning technologies. ${ }^{1}$ For example, in the United States, student enrolment in at least one distance learning course reached $16.7 \%$ in 2016 , while student enrolments in exclusively distance courses reached $14.9 \% .^{1}$

Digital education is rooted in the use of the Internet and web-based technologies, and offers a toolbox that substitutes traditional educational methods, mostly based on the lecture format, where students are passive learners (Dufresne, Gerace, Leonard, Mestre, $\&$ Wenk, 1996). Technological advances create a new scenario, in which new roles, ways of functioning, and priorities emerge for teaching-learning agents. This new approach challenges traditional pedagogic practice by stressing the need for active learners who construct their own knowledge, (Hernández-Lara, Serradell-López, \& Fitó-Bertran, 2019, 2018). Strong online higher education institutions have emerged, and their educational tools have become cornerstones and vehicles for developing a more interactive studentbased teaching-learning process (Sánchez, Campa, \& Hernández, 2011). These educational institutions develop and use communication platforms that promote not only teacher-student and student-student learning interactions, but also teacher-student content interactions (Cheng \& Chau, 2016). This has become an important area for exploration.

Promoting such interactions carries educational benefits as it shifts the focus towards students, who may enrich their learning through applying a constructivist approach, 
take responsibility for their own knowledge construction, and collaborate with their peers (Lonn \& Teasley, 2009).

Interactivity, however, implies major challenges. Firstly, there is some scepticism regarding its unequivocal effectiveness in improving learning (Hernández-Lara, PereraLluna, \& Serradell-López, 2019; Lonn \& Teasley, 2009). Only few studies have addressed this field and their findings have differed. Some studies claim a positive association of interactivity with learning results while, in others, the association is negative (Kent, Laslo, \& Rafaeli, 2016). There are also scholars who find little difference, either in terms of quality or quantity, in the learning results (Picciano, 2002). The literature has reached no consensus on the correlation and impact of student interactivity on learning performance or achievements.

Secondly, interactivity has hitherto been treated from an excessively quantitative view, mainly measuring participation frequency as the number of logins or posts, and participation intensity using the duration or length of interactions (Schellens, Van Keer, Valcke, \& De Wever, 2007). This approach offers a partial view that needs to be complemented with additional information on the quality and contents of student interactivity to arrive at its true value for learning construction (Hernández \& Serradell, 2018; Lonn, Teasley, \& Krumm, 2011). Data analytics and data mining help in accessing the content of student interactions in the course of the learning process, and analysis of the data collected allows us to assess the process in greater depth (Alonso-Fernández, Calvo-Morata, Freire, Martínez-Ortiz, \& Fernández-Manjón, 2019; Alonso-Fernández et al., 2019b). Learning analytics also offer support to teachers who may be unsure about student interactivity and its learning effects, increase teachers' presence in online education, and improve teachers' confidence in acting (Van Leeuwen, Janssen, Erkens, \& Brekelmans, 2015). The application of these techniques is of increased relevance in fields where student evaluation is especially difficult, such as in the case of games - this has fostered the emergence of game learning analytics.

Finally, although much previous research has analysed synchronous and asynchronous learning modes, there is still a need for evidence when it comes to comparing the two in terms of i) communication patterns (Kubasko, Jones, Tretter, \& Andre, 2008), ii) their contribution to students' knowledge and learning performance (Lim, 2008), and iii) the content of the learners' interaction itself. Although online asynchronous interaction has been quite widely researched (Lim, 2008), little is known about the impact of online synchronous interaction or instant messaging, and even less about the different features, content, and performance that emerge when these two learner interaction modalities are compared.

These major challenges motivate the main objective of the present study, which consists in analysing the communication patterns and learning effectiveness related to the use of two basic synchronous (instant messaging apps) and asynchronous communication (online discussion forums) tools, in digital educational environments (specifically business simulation games). This overall objective will be achieved through responding to three research questions:

RQ1: What are the learning results of students using synchronous and asynchronous communication?

RQ2: What are their communication patterns using these tools?

RQ3: What is the content profile of their communication when using these tools?

The comparison between the different types of learner interactions will lead us on to a wider topic, that of student learning results and achievements associated with online education interaction tools. The relevance of this topic lies in the proliferation of online 
and distant learning, as well as the new forms of communication fostered by the Internet, technological advances and social media, and their impact on learning. Analysis of the different features of the communication patterns that characterise synchronous and asynchronous communication tools, as well as the content profile detected in student communications when using both of them, will provide evidence-based recommendations for improving communication in online education, and a better understanding of the impact on learning outcomes.

\section{$2 \quad$ Literature Review}

\subsection{Learning outcomes of learner interactivity}

Interactivity based on digital technology distinguishes between interactions with content (where students and/or teachers upload and access study materials), and social interaction (Paiva, Bittencourt, Tenório, Jaques, \& Isotani, 2016) between the agents of the teachinglearning process, such as teacher-student or learner-learner interactions (Hernández-Lara et al., 2019; Rachman-Moore \& Kenett, 2006). With the advent of new technologies and a higher complexity of online education, other forms of interactivity have emerged such as interactions with the online interface itself and self-interaction (Paiva et al., 2016).

Social constructivist theories of education highlight the role of social interactions in promoting knowledge construction (Karahan \& Roehrig, 2015; Kent et al., 2016) and note that the connection between interactions and learning will depend on the interaction characteristics. This relationship emerges as much as interaction impacts include engagement, teamwork, analysis, coordination, negotiation, problem-solving, and reflection, all of them relevant ingredients for sharing and constructing knowledge (Boticki, Baksa, Seow, \& Looi, 2015; Kent et al., 2016).

The study of the effects of learner interactivity at an educational level has attracted the attention of previous research, but there is no clear consensus on its impact on student learning results (Adeyinka \& Abdulmumin, 2011; Picciano, 2002; Song \& McNary, 2011; Wei, Peng, \& Chou, 2015). Some studies have highlighted a positive link between learner interactivity and results. These include Wei et al. (2015) who pointed out that students with frequent access to an online platform and with more interactions showed improved learning. This positive association could be explained by the impact of learner interaction on their satisfaction and the creation of learning communities with positive effects on learning outcomes (Chaparro-Peláez, Iglesias-Pradas, Pascual-Miguel, \& Hernández-García, 2013). Boticki et al. (2015) also found that the quantity and quality of student interactions were related to better exam results. Likewise, Yueh, Lin, Liu, Shoji, \& Minoh (2014) noted that both instructors and learners agreed on improved interaction leading to a better sense of a learning community, a reduction of isolation, and an improved performance. These results are similar to those of Wang \& Walker (2013), who remarked that collaboration increases engagement and, in science and engineering, is correlated with greater learning achievements.

Other studies, however, have opposing findings. For example, in their study on online discussion posts, Song \& McNary (2011) found multiple topics in student conversations and no correlation between the number of posts and overall student marks. This result was provoked by finding that the posts' content was frequently unrelated to the 
specific learning objectives of the course. Picciano (2002), also found an inconsistency between the posting son discussion boards and performance related to the courses' goals, although the reasons behind these findings were not explored. More recent studies emphasise this lack of connection between learner interactivity and either the students' perception of their learning (Chaparro-Peláez et al., 2013) or competences such as commitment and teamwork (Iglesias-Pradas, Ruiz-De-Azcárate, \& Agudo-Peregrina, 2015). These findings may be justified in various ways, such students sometimes preferring more individual, self-directed learning styles, or their perception of interaction as an inherent element of how they learn (which reduces its relevance as a predictor of perceived learning).

These studies also highlighted the excessive relevance given to quantitative measures in describing the characteristics and functioning of interactivity. The most-used metrics were number of logins, posting frequency, number of read messages, and messages lengths (although other metrics also exist). This quantitative approach measures learning performance mainly by course marks. Finally, because computer-mediated communication can be easier checked, designed, and controlled, these studies mostly dealt with asynchronous interaction based on communication tools (such as online discussion forums or similar methods), rather than in synchronous communication or instant messaging (Lim, 2008). Asynchronous communication also implies a form of learning that fosters reflection and communication and is not time-dependent, with consequent spatial and temporal flexibility benefits (Kim, Yoon, Jo, \& Branch, 2018).

These inconsistent findings and gaps encourage further study on synchronous tools of communication and a continued exploration of the impact of learner interactions on their learning which should also include qualitative measures of interaction and learning achievement.

\subsection{The effects on learning of synchronous and asynchronous communication tools}

Interaction in online learning revolves around a variety of communication modes (Madden, Jones, \& Childers, 2017) but, basically, these can be classified into synchronous and asynchronous (dos Santos \& Cechinel, 2019). Synchronous means working together at the same time, frequently teacher-paced using, for example, instant messaging or chats, online conferences, software for user desktop sharing, etc; while asynchronous involves a time delay between the release and the reception of information, such as in the case for discussion forums and emails (Alario-Hoyos et al., 2018). Both learning modes not only affect communication but other aspects that imply transmitting and delivering the necessary information for the teaching-learning process (Hampton, Pearce, \& Moser, 2017).

Previous research on both types of interaction at an educational level has been focused on better understanding their characteristics, roles, and effects (dos Santos \& Cechinel, 2019). Scholars have generally considered the two types as complementary (deNoyelles \& Raider-Roth, 2016; Muniz-Solari \& Coats, 2009), because both support collaboration. Mostly, they are considered together, without remarking on the differences between them and their different purposes (Meyer, 2015; Wang \& Walker, 2013; Yueh et al., 2014). A few studies, however, do contrast their differences and suitability.

Some of these studies have emphasised the different characteristics and features of communication patterns in synchronous versus asynchronous mode. For example, the study conducted by Alario-Hoyos et al. (2018) analysed learning interaction tools when running MOOCs in terms of time spent and accesses, found that in the case of synchronous modes the use of the tool is concentrated in shorter periods of time but the accesses 
to the tool were higher. Similarly, Sere et al. (2011) point out that a higher percentage of social interactions occurs in synchronous rather than asynchronous modes, while students spend more time in task-oriented interaction in asynchronous discussions.

Analyses of the preference for one specific form of communication over the other, sometimes explained by the user type, are frequent in the literature. For example, dos Santos \& Cechinel (2019) noted that, although both forms received good ratings assessments during the final year project supervision in a distant learning course, students and supervisors preferred asynchronous forms of communication. Hampton et al. (2017) studied the preferences of students and teachers in online nursing courses by considering different modes of synchronous and asynchronous tools (videos, narrated PowerPoint presentations, Adobe Connect education sessions, article readings, e-mails, wikis, simulations/games and case studies). They found that the most engaging and effective method depended on the students' generation (Baby Boomer, Generation X or Millennial). Smithson, Jones, \& Ashurst (2012) demonstrated in their study on mental health system that synchronous formats of participation encouraged the users more, while professionals showed a preference for asynchronous formats.

Although the characteristics and features of interaction, as well as user satisfaction and preference are important, they do not directly predict outcome success in terms of learning (Nowak, Watt, \& Walther, 2009). Most previous analyses of learning outcomes of synchronous and asynchronous interactions are of the latter (Kim et al., 2018; Lim, 2008). Few studies have analysed synchronous modes or have conducted comparison studies between them. A notable exception is the study of dos Santos \& Cechinel (2019), who considered effectiveness in terms of appropriateness and sufficiency of both forms of communication for different types of discussions. They concluded that both forms were appropriate and sufficient for the discussion of academic and relevant topics of a final year project, but they worked better for more experienced students, while less experienced ones preferred face-to-face meetings rather than online communication. Korkmaz (2013) compared both forms of interactions in terms of their influence on learner attitudes towards the Internet and web-based learning, as predictors of their learning results. He remarked that asynchronous interaction made a significantly higher contribution to learner attitudes towards web-based learning, while no differences were detected between synchronous and asynchronous regarding learner attitudes towards the use of the Internet. Asynchronous interaction was preferred in text-based chat platforms when any kind of language problem existed, like the compulsory use of a language different from the mother tongue. The study of Santoveña-Casal (2012), based on synchronous communication tools emphasized that they reinforce the professional, personal and socio-emotional relationships of participants, which potentially could impact on cognitive and knowledge construction. Lim (2008), in a study on the impact of chat interaction on knowledge construction processes, showed that this tool improved participants' involvement in meaning negotiation, as well as the availability of scaffolding and shared understandings, which are characteristic of knowledge construction processes. Finally, Davidson-Shivers, Muilenburg, \& Tanner (2001), in their study comparing chats and discussions forums, found that substantive communication (directly related to the topic) was higher when using chats than when using discussion forums, as well as marks - a finding confirmed by the study of Johnson (2008) on students of an educational psychology course. 
Previous research has emphasised advantages to both synchronous and asynchronous interaction, although the overall picture is unclear because the benefits and shortcomings of each communication form could be different depending both on the participants, and on the targets considered to determine whether the learning outcomes really occur. In summary, more evidence is needed before we can reach a holistic view of the contribution of both types of interactivity. Findings to date need to be complemented by quality and quantity indicators of both types of learners' interactions, in order to better determine, qualitatively and quantitatively, the profile, roles and effect of these different communication modes.

Our general objective recommends the use of an approach largely neglected by previous research and focuses on analysing the content of students' discussions when using synchronous and asynchronous communication, through learning analytics techniques and data mining on natural language processing. This can improve the evaluation of students' learning process, allowing educational institutions to make more informed decisions (Alonso-Fernández et al., 2019a). These techniques can also be applied to games which are characterised by providing an appropriate environment for interaction and for data-capture (Alonso-Fernández et al., 2019b). The application of game learning analytics is a mechanism for overcoming the difficulties of assessing student participation in games, allowing access to more information related to the learning process of players which is not evident using traditional assessments (Lee, Donkers, Jarodzka, \& van Merriënboer, 2019).

Our study aims to contribute to the field of game learning analytics, adding evidence on the role of synchronous and asynchronous communication tools for the learning improvement of students participating in games.

\section{Methodology}

\subsection{Data collection}

The participants of our research were students of the Universitat Oberta de Catalunya, an online higher education institution of Spain, who were studying business and management courses at bachelor level. Data from seven academic years were retrieved (20112012 to 2017-2018 inclusive). The selected students were participants in the non-compulsory course "Business Simulation and Practice". This was a 4 month-long course offered in the first and second semesters of the academic year. The course required the participation of students in a business simulation game. The game chosen was Cesim Global Challenge (www.cesim.com), a strategy game where student/player teams have to manage a simulated international telecommunication enterprise. The game provides an experiential learning scenario based on global businesses and the operations of management-related functional areas (demand, production, marketing, human resources, logistics, research and development, taxes and finance), and emphasizes the interrelationships between them, while developing generic competences in decision-making, teamwork, and problem solving.

The game consisted of eight rounds, one a week, the first two being practice rather than competitive (not considered for the calculations of the economic and financial performance of the simulated companies). The course also included three weeks when students did not play but worked in the reports used for course assessment.

The game was played completely online, without face-to-face interactions among players, which promoted the use of different interactivity modes. The game provides an online discussion forum which fosters student interaction, records their contributions and 
messages. Some teams felt very comfortable using this communication tool and considered it useful and efficient to record their communication during the game. However, other teams preferred different forms of interaction, because the discussion forums were not considered fast or agile enough to facilitate their participation in the game (Hernández \& Serradell, 2018; Hernández-Lara et al., 2019).

For the purpose of this research, the teachers fostered the interaction of students within their teams by promoting a single communication. Only one communication mode was offered to each course, and students chose voluntarily whether or not to participate. These communication tools were either asynchronous, using the online discussion forum provided by the game; or synchronous, using an instant messaging app. Specifically, the teachers decided to use Telegram, which is a free cloud-based instant messaging service whose the client-side code is open-source software. Telegram allows creation of a Telegram Bot access and record the communication within the teams using this tool. The students were informed about this, and whether or not to participate in the experiment, using the instant messaging app, the online discussion forums, or none of them, knowing that no personal data would be used and that all the information retrieved would be treated anonymously and only for academic research purposes. In all cases, student interactions occurred without any intervention of the teacher.

Teachers encouraged the participation of the students and emphasised its relevance as the only way that they could have access to the online student interaction. To motivate students to take part in the experiment, they argued that these communication tools provide information about their involvement and dedication, which is useful for properly evaluating their performance during the course. The participation ratio of the students was very high, especially in the courses where the interaction mode proposed was the instant messaging app, which exceeded $94 \%$, and reached $81.9 \%$ in the courses where the communication mode was the online discussion forum. The Mann-Whitney U test confirmed that there were no significant differences in the grade levels of students who decide to take part or not in the experiment $(\mathrm{U}=6.876$, p value $=.562)$, as well as in the main financial indicator of the game, the total cumulative shareholders' return $\left(\chi^{2}=\right.$ $11.367, \mathrm{p}$ value $=.854)$.

From a total population of 551 students, the final sample was composed of 478 (267 using online discussion forums and 211 using the instant messaging app); distributed among 106 teams (63 using online discussion forums and 43 using the instant messaging app); and playing in 13 different competitions ( 9 in the case of the online discussion forums and 4 in the case of the instant messaging app). Teams were composed of an average of 4.24 members in the case of the courses using online discussion forums (ranging from 2 to 6 students), and an average of 4.9 members in the courses where the instant messaging app was used (ranging from 4 to 6 students). Likewise, 213 students were female (44.56\% of the sample, 117 in the online discussion forums courses and 96 in the instant messaging app courses) and 265 were males $(55.44 \%$ of the sample, 149 in the online discussion forums courses and 116 in the instant messaging app courses). The average age of students was 32.43 years. 


\subsection{Measurement of variables}

Learning outcomes and results were measured through quantitative indicators in a first phase, which included their grades or marks (Marks) in the course that integrates the business simulation game, as it has been frequently reported by previous research (Boticki et al., 2015; Kent et al., 2016). We also measured the economic success of the player teams in the game, considering operational and financial key performance indicators, as follows: earnings per share (EPS), the total cumulative shareholder return (TCSR), and profit at the end of the game (Profit). The winners were the teams with higher TCSR, which is an indicator that includes the change in the share value, the dividend paid out to shareholders and the profitability of these dividends over time.

The 478 students of the sample posted a total of 39,503 messages. The complete text corpus was composed of 560,123 words, with an average of 14.18 words per message in an unstructured format. The analysis of the content of students' conversations was conducted using natural language processing. Since Catalonia is a bilingual region, where Catalan and Spanish coexist as official languages, messages might be in either Catalan or in Spanish. Natural language processing (NLP) included pre-processing the corpus, stripping white space, conversion to lower case, elimination of stopwords (Rajaraman \& Ullman, 2012), removal of punctuation and stemming (suffix stripped, (Porter,1980); R tm v.0.6e2 (Feinerer, Hornik, \& Meyer, 2008). A document term matrix (DTM) was constructed with the pre-processed corpus, including the frequency of terms per forum and instant messaging interactions in a sparse matrix that represents the corpora. The DTM was subject to a sparsity filter $(\rho>8)$ and normalized to the number of occurrences per message. R version 3.3.2 (R Core team, 2018) was used to conduct the NLP, specifically the tm R package (v. 0.6e2, (Feinerer, Hornik, \& Meyer, 2008)).

\section{Data Analysis}

\subsection{Learning results using synchronous and asynchronous communication}

In order to respond to the first research question, that explores the quantitative learning results of students using synchronous and asynchronous communication when participating in business simulation games, we conducted mean comparison analyses of the learning outcomes and results achieved by students (Marks, TCSR, EPS, Profit).

These learning outcomes were not normally distributed, as the Shapiro test confirms in all of them $(\mathrm{W}=0.96$, $\mathrm{p}$-value $<0.001$ for Marks; $\mathrm{W}=0.79$, $\mathrm{p}$-value $<0.001$ for TCSR; W=0.91, p-value $<0.001$ for EPS; $\mathrm{W}=0.89$, p-value $<0.001$ for Profit). This implies the use of the non-parametric Mann-Whitney $U$ test to conduct the mean comparison analyses between both groups, students using online discussion forums and the instant messaging app. This test determines if two independent samples are selected from populations with the same distribution. Results are displayed in Table 1.

\section{[Insert Table 1]}

From Table 1, we can observe that significant differences exist in the mean value of all the quantitative learning results related to the Marks obtained by students in the course, as well as the economic and financial key indicators of the game (TCSR, EPS and Profit at the end of the game). These results are higher in the case of students using asynchronous communication through online discussion forums, who outrank the students using synchronous communication in all the indicators. 


\title{
4.2 Communication patterns in synchronous and asynchronous communication tools
}

To respond to the second research question, that explores the different features of the communication patterns that characterised synchronous and asynchronous learner interactions, we extracted some descriptive statistics. Table 2 exhibits characteristics of the communication patterns of students using the online discussion forums and those using the instant messaging app, in terms of messages, words, and words per message.

\section{[Insert Table 2]}

These basic data from Table 2 show that the total number of words used by students in their interactions with both tools were almost the same, however the number of messages in the app was much higher than that in the online discussion forums (almost 9 times more). This finding implies that the number of words per message was higher in the online discussion forums than in the instant messaging app. That confirms expected results, such as the longer length of the messages in the forums in comparison with instant messaging.

The histograms for the online discussion forums (Figure 1) and the instant messaging app (Figure 2), evidence their different distribution in the length of the messages (number of words per message). The interaction with forums is characterized by few short messages, this number grows when the number of words also enhances to decrease afterwards, as the number of words continues to grow. In the interaction with instant messaging, the most frequent situation is messages with very few words, very few long messages (for example, messages over 100 words are almost non-existent) and a high number of messages with a similar length.

\author{
[Insert Figure 1] \\ [Insert Figure 2]
}

In addition to the number and length of messages, the time aspect of the communication pattern is relevant. Our analysis allowed us to explore, for example, when communication took place, the change in interaction over the course of the game, as well as the concentration of communication at specific time points, and the time between responses. To conduct these analyses, we used the start time of each message as given by the forums and instant messaging tools.

Figure 3 and 4 exhibit the time of day at which communication takes place, for the online discussion forums and the instant messaging app, respectively.

\section{[Insert Figure 3] \\ [Insert Figure 4]}

The distribution of the number of messages depending on the hour of the day shows that the communication of students participating in the simulation online took place mostly in the evening (after 8 p.m.). The distribution also shows that interactivity in the sample of students using the online discussion forums during the morning (since 
10 p.m.) and in the afternoon (after 3 p.m.) was also frequent, but in a lesser extent, the bias towards night messages being larger in the case of instant messaging than in the case of forums.

Figures 5 and 6 show the communication pattern of students throughout the course, for the online discussion forums and the instant messaging app, respectively. The distribution of the messages over the weeks in each term (first and second semesters) was considered. This allows us to see whether student interaction depended on the time point within the course. The split of Figure 5 into two separate distribution graphs means that students using online discussion forums participated in the game during the whole academic year (in courses that took place in both, the first and second semester); while Figure 6 indicates that students using the instant messaging app always belonged to courses that took place only in one semester.

\section{[Insert Figure 5] \\ [Insert Figure 6]}

From the Figures above, it can be concluded that the interactions were not homogenously distributed throughout the terms, and depended on the week the communication was higher or lower, meaning that during the game, there were moments in time that merited increased communication. It is clear from the figures that the communication distributions differ depending on the tool used. For example, students using online discussion forums interact more during the first weeks of the game, when their knowledge and experience of the software, tasks and work processes involved in the simulation are lower. On the other hand, students using instant messaging show high peaks of communication until the middle of the course, with two high peaks coinciding with the first and second quarter, and this decreases during the last half of the course.

Finally, Figures 7 and 8 exhibit the concentration of messages over time, when students used online discussion forums and instant messaging, respectively. It gives an idea of the time that elapses between messages, measuring the minutes between them to discover whether or not players respond immediately.

\section{[Insert Figure 7]}

[Insert Figure 8]

These Figures point out that a high number of messages was immediate in the two samples. However, while when using the app, the majority of messages occurs within the first minute, and this number decreases when time goes by; among students using online discussion forums the number of responses remains more or less the same as time passes, the decrease in the number of messages over time being lower than in the instant messaging app sample. Only after a very considerable time lapse (for example 1,000 minutes, around 16 hours), is there a noticeable decrease in the number of messages in the sample using the forums.

\subsection{Communication contents in synchronous and asynchronous tools}

The third research question is focused on analysing the content profile of the students' communications when using both synchronous and asynchronous tools.

To respond to this question, some exploratory analyses were conducted. Firstly, we show the most frequent stems in the text corpora of both tools. Table 3 displays their absolute count in the corpus of the sample using online discussion forums and the instant messaging app, respectively. 


\section{[Insert Table 3]}

The analysis in Table 3 evidences the sparsity of the stems (higher in the case of instant messaging), which means that very many stems have a low frequency of occurrence. The findings also show considerable overlap between the most repeated stems in each communication tool. Another relevant conclusion is that most of the frequent stems are task-oriented, and only a few refer to other communications, such as salutations ("hola" or "saludos" would be good examples, as the Spanish words for hello and goodbye).

Some of the most frequent stems referred to concepts related to the core of the game. This is the case for "tec" (abbreviation for technology), which refers to the industry of the simulated companies, this being the most frequent stem in both samples $(5,806$ times in the forums and 2,384 in the app). Other frequent stems that point in the same direction are: "mercado" ("market" in Spanish that appeared 2,625 times in the forums), EEUU (1,828 times in the forums and 705 times in the app), Asi $(2,203$ times in the forums and 1,253 in the app), Europ (1,463 times in the forums and 510 in the app), which are Spanish stems for the three geographical areas that constitute the international markets in the game); "preci" or "precio", price in Spanish (2,572 times in the forums and 578 in the app); "demanda" or "demad" as one of the main functional areas requiring in-game decisions (1,796 times in the forums and 502 in the app); or economic and financial terms related to the performance of the simulated companies, like "beneficio" ("profit" in Spanish appearing 772 times in the forums); "cuota" ("market share" in Spanish, 1,121 times in the forums); or "resultado" ("outcome" or "result" in Spanish, 1,090 times in the forums).

Besides the concepts that illustrate the content of the game, other frequent stems referred to its purposes. This happens for example with the stem "decisión" (2,340 times in the forums and 457 in the app); or characteristics of the course like pac or pec (the names used to refer to the reports delivered by students for their assessments and marks, which appeared 1,092 times in the forums and 500 times in the app), or "grupo" and "equipo" (the Spanish words for team, i.e. the way players participate in the game, appearing 900 times in the forums).

There are also frequent stems referred to time, like "ahora" ("now" in Spanish), which relates to time-oriented issues of the game. Finally, the most frequent verbs in the conversations express most of the time uncertainty, which is another relevant feature of the game related to competition and the uncertain changes in the markets and the global environment, as evidenced by of "parec" or "sembl" (it seems), "pued" (it could be) and "cre" or "crec" (I think).

After indicating the most frequent content of the students' conversations, we explore if some kind of relationship exists between the most frequent stems and concepts that appeared in the students' online interactions. To confirm these relations, we calculated the bivariate correlation between the most frequent terms per message. This analysis is exhibited in Figures 9 and 10, for 40 of the most frequent stems in the online discussion forums and the instant messaging app respectively.

\section{[Insert Figure 9]}




\section{[Insert Figure 10]}

The correlograms show that, considering the correlations between the most frequent stems, some differences are observed between the online discussion forums and instant messaging. Higher, stronger and positive correlations between frequent stems were found in the sample using the online discussion forums, in comparison with the sample using the instant messaging app, where the correlations were fewer, weaker and less positive, in general. Both correlograms allow us to conclude that the most frequent and related contents, were not exclusive and were game-oriented to the tasks, purposes and characteristics of the game.

Stems like "tec" (technology), "cuot" (market share), "EEUU" (USA), "Asi" (Asia), "Europ" (Europe), "producción" (production), "benefici" (profit), "mercado" (market), "grupo" (team), "empresa" (company), "característica" (technology features), "venta" (sales), "coste" (cost), "precio" (price), "acuerdo" (agreement), "resultado" (outcome), "ronda" (round), "decisión" (decision), "mejor" (better), etc. are positively and strongly related in the sample using online discussion forums

In the interactions with the instant messaging app, the stems related were: "demand" (demand), "Asi" (Asia), "Europ" (Europe), "EEUU" (USA), "vend" (sales), "tec" (technology), "preci" (price), "buen" (good), "parec" (to seem), "bien" (well or fine), "pod" (to can), "hac" (to do), "pon" (to put), "pued" (to can), "cre" (to think), "decisión" (decision), "merc" (market), "cost" (cost), among others. It can be seen that the correlations in this case were not so strong, and not always related to the core concepts of the game (conversations were more disperse using the instant messaging app than online discussion forums), and that some negative correlations also appeared, for example between the stems used in Spanish and Catalan, which means that students who normally use one language do not use the other.

From these analyses another relevant finding emerges, as a consequence of the use of Spanish and Catalan in the interactions. Even although it might be considered as a limitation (as we discuss later), the reality of the overlapping use of the two official languages allows us to explore the communication pattern under this specific circumstance. For example, the major frequency of Spanish stems shows the preference of students for using this language in their communications while participating in the game. Spanish constitutes the self-selected language after the first interactions within the team, because if anyone in the team begins speaking in Spanish, the other team members reply using this language (whose use is more generalised), in order to facilitate the communication. This preference is more evident in the case of online discussion forums than in instant messaging, where the communication is more spontaneous. The results provided by the application of NLP are still reliable because the use of Catalan was residual and due to the great similarity between roots in Catalan and Spanish words.

\section{Discussion and Conclusions}

The principal objective of this study consists in analysing the effectiveness of two basic synchronous (an instant messaging app) and asynchronous communication tools (online discussion forums) used in digital education, specifically in the case of business simulation games. The purpose is to gain a better understanding of the students' learning results and achievements associated with their communication tools, the different features of their communication patterns, and the content profile of their interactions when using these different tools. 
With regards to the first research question, which explores the quantitative learning results of students using synchronous and asynchronous communication when participating in business simulation games, the results confirm that marks as well as economic and financial key indicators of the game were higher in the case of students using asynchronous communication - these outrank the students using the instant messaging app This finding agrees with previous studies that found a positive impact of learner interactivity on learning results in online discussion forums, as a result of higher satisfaction, the construction of learning communities, and a reduction in isolation in the learning process (Boticki et al., 2015; Chaparro-Peláez et al., 2013; Wei et al., 2015). Few studies have conducted comparative analyses between the two forms of communication, and even fewer have specifically compared the learning results achieved with them. Among such studies as do exist, there is no clear consensus, but some scholars have remarked on the benefits of asynchronous tools over synchronous ones in terms, for example, of the attitudes of student towards web-based learning (Korkmaz, 2013), or when any difficulties relating to language skills exist. As opposed to other studies which highlighted the positive impact of synchronous communication in terms of social relationships and negotiation (Lim, 2008; Santoveña-Casal, 2012), in our case, we found that the impact on learning results was greater when asynchronous tools of communication were used.

With regards to the second research question, regarding the description of the different communication patterns that emerge using both tools, our findings highlight that they are really different. The number of messages in the instant messaging app was much higher than in the online discussion forums, and their length (number of words) was lower. Also, there was a high number of messages of a similar length in the app. These findings are not surprising and are in line with those of previous studies which analysed the time spent and number of accesses using synchronous and asynchronous tools and which concluded that synchronous modes showed more accesses (higher number of messages), were more concentrated in time (Alario-Hoyos et al., 2018), and were linked to a higher percentage of social interactivity (Sere et al., 2011).

However, our study takes a step further by analysing in depth the communication pattern over time and extracting some relevant conclusions. For example, in terms of the time of day when communication occurs, the bias towards night messages was larger in the case of the app than in the case of forums. A possible reason for this might be related to the different devices normally used in each communication form, usually computers for the online discussion forums, and mobile phones for instant messaging. Communication via mobile phone facilitates interactions at any time, especially in the evening or at night, when students have more time after finishing other work. In addition, interactions are not uniform throughout the term. Students using online discussion forums interact more during the first weeks of the game, when their knowledge and experience of the software, tasks and work processes are lower. On the contrary, students using instant messaging show higher peaks in communications during the first half of the game. This result points out that the greater agility of instant messaging fosters the students' interactions over longer periods. Finally, although a shorter time lapse between messages might be expected in instant messaging, our findings confirm that messages were quite immediate in both cases, but among students using online discussion forums the number of responses remains approximately constant, only dropping after very considerable amount 
of time has elapsed. This finding confirms the idea of time-pressure as a more relevant feature of instant messaging, compared to discussion forums.

Finally, regarding the third research question, which refers to the content analysis of communication using synchronous and asynchronous tools, our findings note the sparsity of the stems, a great overlap between the most repeated stems in the two communication tools, and the fact that most of them referred to concepts that constitute the core of the game (such as the industry of the simulated companies, the purpose of the game, their main functional areas, and economic and the in-game financial indicators). Stems relating to time management and uncertainty were also frequent. This content schema was similar to that of previous studies on business games (Hernández-Lara et al., 2019; Hernández \& Serradell, 2018), and appeared in both communication tools. In addition, these concepts were highly correlated, appeared together, were not exclusive, and were task-oriented in line with the purposes and characteristics of the game, as previous studies on online discussion forums also showed (Hernández-Lara et al., 2019; Sere et al., 2011). However, the correlations between the most frequent stems when interactions take place with the instant messaging app were not so strong, and sometimes did not emerge between stems related to the core concepts of the game, which means that conversations using this tool were more disperse and not so game-oriented as in online discussion forums, as previous studies had already suggested (Sere et al., 2011).

The negative correlation between the stems used in Spanish and Catalan means that the students who normally using one language do not use the other. The major frequency of Spanish stems shows the preference of students for using this language in their in-game communications. It also implies that in this bilingual region, where both languages are used indifferently, when a specific language is first used, it quite frequently continues to be used throughout the conversation.

In regard to research, our results improve current knowledge on the similarities and differences of the communication patterns developed by students when they use online discussion forums and instant messaging apps. Specifically, our results take a step forward in analysing in depth the communication pattern over time, in terms of the time of day when communication takes place, over the term of the academic course, and in considering the elapsed time between messages.

We also learn that the effectiveness of the two communication tools in terms of learning differs. From a quantitative perspective, the student learning results were higher when using online discussion forums. From a qualitative perspective, we note the sparsity of stems, a great overlap between the most repeated stems, which were mostly referred to the core of the game: the industry of the simulated company in the game, its purpose, functional areas, and economic and financial indicators; as well as terms related to time management and uncertainty. These concepts were highly correlated, especially in the communication with online discussion forums.

All these findings enrich previous knowledge, on the contribution of students interactions to their learning, on the differences of these contributions depending on the tool used for this communication, and the suitability of learning analytics for exploring student interactions, usually unavailable to teachers, which thus allow them to obtain a fuller picture of the learning process.

\subsection{Contributions}

The findings of this study make some important contributions; these constitute the added value of our work. We provide evidence on the role and contribution of different communication tools, synchronous and asynchronous, used by students who take part in business simulation games. This research contributes to the field of game learning analytics, where 
the nature of games, results in player conversations are not always being focused on learning contents. This explains the mixed results on the influence of interactivity on in-game learning, making worthwhile the comparison of conversation content when players use different communication tools.

More specifically, from a theoretical point of view, this research reinforces the assumptions of social constructivist educational theories underlining the connection between interactivity and learning. Our results confirm their main assumption regarding the influence of learner interactivity on learning results. But our findings take these theories a step further by supporting the idea that the effects of learners' interactivity depend on the tool used, on the communication pattern that characterises each tool, as well as on the contents usually exchanged.

From an empirical perspective, this study reveals the usefulness of learning analytics techniques in gaining insight on learner communication content and the students educational experience through a better assessment and understanding of their learning process, which might be lacking for teachers in online education, either because of lack of awareness of the interactions among students and the consequent effects on learning (Hernández-Lara et al., 2019) or because of information overload that hampers teachers' attention (Van Leeuwen, Janssen, Erkens, \& Brekelmans, 2014). Even more, the application of learning analytics is especially valuable in the case of business simulation games, due to their open-ended nature and the multiple behaviours developed by students while playing, which makes remarkably difficult to assess student learning through traditional measurements (Lee et al., 2019).

This study makes also relevant contributions for practitioners, especially teachers and educational institutions in online environments, such as online higher education institutions, suggesting the suitability of implementing communication platforms that foster and record student interactions. Due to the complementarity of different communication tools, these platforms could be both synchronous and asynchronous, asynchronous tools being especially suitable for task-related communication and synchronous ones for speeding up and facilitating interaction among online students. Our results also suggest the suitability of applying learning analytics to the information collected from student interactions to gain a better knowledge of their learning process and better inform the decisions of teachers and educational institutions.

\subsection{Limitations}

Of course, our research is not exempt from limitations. Firstly, we have the added complexity of the use of different co-official languages in the study region. Even although the use of Catalan was limited compared to that of Spanish in this experiment, and even if the roots of Spanish and Catalan words are very frequently the same, the mix of both could influence the absolute count of frequent terms, hiding the real frequency of some terms that appear in both languages. We addressed this by analysing and grouping the language-independent meaning of the most frequent stems, but it might be advisable to repeat the content analysis using NLP after all the students' interactions are translated into Spanish. Due to the high volume of messages incorporated in this study, this would be challenging. But even with the data that we collected, and considering both languages, 
relevant conclusions emerged, specifically related to the communication patterns of bilingual students, that could not have been detected if all the messages had been translated in the first place.

It is also necessary to mention that this study has focused on business simulation games, to the exclusion of other educational computer systems. This specific focus places the contributions of this study in the field of game learning analytics (Alonso-Fernández et al., 2019b). The application of learning analytics to interactions in other educational computer scenarios, such as learning management systems or massive open online courses, could offer fuller knowledge on the contribution of student interactivity to the learning process, and the specific role played by different communication tools.

Another relevant limitation is the exploratory nature of this study which encourages further explanatory analyses, considering the learning results as a consequence of the communication developed by students. This more advance research will allow us to determine which is the most efficient communication tool in terms of the student learning achievements. It constitutes an interesting challenge for the future research agenda. 